\title{
FATORES A SEREM CONSIDERADOS NA UTILIZAÇÃO DE BEAUVERIA BASSIANA VISANDO O MANEJO DE POPULAÇÕES DE ALPHITOBIUS DIAPERINUS EM AVIÁRIOS COMERCIAIS
}

\author{
L.F.A. Alves ${ }^{1 *}$, P.M.O.J. Neves ${ }^{2 *}$, R.C. de Oliveira ${ }^{2}$, D.G.P. de Oliveira ${ }^{1 * *}$ \\ ${ }^{1}$ Universidade Estadual do Oeste do Paraná, Centro de Ciências Biológicas e da Saúde, Laboratório de Zoologia \\ de Invertebrados, Rua Universitária, 2069, Cascavel, PR, Brasil. E-mail: 1faalves@unioeste.br
}

\section{RESUMO}

\begin{abstract}
O trabalho foi desenvolvido com o objetivo de comparar a eficiência de preparações líquida e em pó do fungo Beauveria bassiana e também verificar quais efeitos que o tipo de solo e cama de aviário podem causar sobre o fungo, em condições de laboratório. A formulação em pó mostrouse mais eficiente que a líquida, causando mortalidade confirmada de larvas de 83,3 e de 33,4\% de adultos, contra 18,7 e $12 \%$, respectivamente para larvas e adultos. $\mathrm{A} \mathrm{CL}_{50}$ da preparação em pó foi de $2,41 \times 10^{7}$ e 5,33 × $10^{7}$ conídios/g e $\mathrm{TL}_{50}$ de 4,7 e 5,3 dias para larvas e adultos, respectivamente. O fungo em contato com o solo de aviário teve sua eficiência reduzida em cerca de $40 \%$ em relação ao tratamento com solo de barranco, e a mortalidade de larvas não diferiu quando se utilizou cama nova ou usada. A mortalidade de adultos foi baixa tanto para solo combinado à cama nova $(11,5 \%)$ quanto usada (25\%), bem como a mortalidade de larvas na aplicação do fungo sobre $(58,7 \%)$ e abaixo $(38,7 \%)$ da cama nova. Constatou-se que o aumento do tempo de contato dos insetos com os substratos tende a diminuir a mortalidade em cerca de 12 vezes, independente do substrato.
\end{abstract}

PALAVRAS-CHAVE: Estratégia de aplicação, cascudinhos, aviário, frangos de corte.

\section{ABSTRACT}

FACTORSTOBE CONSIDEREDINTHEUSEOFBEAUVERIA BASSIANAFORTHECONTROL OF ALPHITOBIUS DIAPERINUS ON POULTRY FARMS. This study was carried out in order to compare the effectiveness of liquid and powder preparations of the fungus Beauveria bassiana, and to verify the potential effects of soil type and poultry farm bedding on the fungus, under laboratory conditions. The powder proved more effective than the liquid formulation, causing a confirmed larval mortality of $83.3 \%$ and a confirmed adult mortality of $33.4 \%$, against 18.7 and $12 \%$, respectively, for larvae and adults. $\mathrm{LC}_{50}$ values of $2.41 \times 10^{7}$ and $5.33 \times 10^{7}$ conidia/g, and $\mathrm{LT}_{50}$ values of 4.7 and 5.3 days were obtained with the powder preparation for larvae and adults, respectively. When in contact with the poultry farm soil, the fungus had its effectiveness reduced by about $40 \%$ compared to the hillside soil treatment, and no differences in larval mortality were observed between fresh and soiled bedding. Adult mortality was low in the soil combined with either fresh $(11.5 \%)$ or soiled bedding $(25 \%)$, as was larval mortality in the application of the fungus over $(58.7 \%)$ or below $(38.7 \%)$ the fresh bedding. It was verified that an increased contact time of the insects with the substrates tended to decrease mortality by about 12 times, regardless of substrate.

KEY WORDS: Application strategy, lesser mealworms, poultry farm, broiler chickens.

\section{INTRODUÇÃO}

Alphitobius diaperinus Panzer (Coleoptera: Tenebrionidae), conhecido como cascudinho, é um inseto comum em aviários de postura e corte, onde encontra abrigo, alimento em abundância e ausência de competidores e/ ou predadores (GeDENetal.,1998; Chernaki; Almeida, 2001a; Chernaki-Leffer, 2004).

Seus danos causam aumento nos custos de produção e preocupações ao setor avícola devido ao perigo potencial destes insetos na transmissão de vírus, bactérias, fungos e protozoários. São também utiliza-

\footnotetext{
${ }^{2}$ Faculdade Assis Gurgacz, Cascavel, PR, Brasil.

*Bolsista de Produtividade em Pesquisa, CNPq.

**Bolsista de Iniciação Científica, CNPq/PIBIC.
} 
dos como alimento alternativo pelas aves, ocorrendo diminuição do peso em função da redução da conversão alimentar, e pelas lesões no trato digestivo e as aves quando buscam os insetos, inalam poeira da cama, sofrendo irritação do trato respiratório (STEINKRAUS et al., 1992; Despins et al. 1994; Despins; AxTelL, 1995).

Atualmente, para o controle do inseto, utilizam-se inseticidas químicos de curto período residual, aplicados na superfície da cama ou das paredes que, devido ao ciclo de vida curto e hábitos crípticos do inseto, são ineficientes e permitem reinfestações (Axtell; Arends, 1990). O manejo da cama também pode oferecer bons resultados (CHERnAKI; ALmeIdA, 2001b), apesar de trabalhoso.

Diante da necessidade de implantação de medidas mais eficazes e menos perigosas do ponto de vista da saúde dos produtores e consumidores, estudos realizadosnosEUA, etambémnoBrasil, demonstrama presençadefungosentomopatogênicosnosaviários(STEINKRAUS et al., 1991; Alves et al., 2004; Alves et al., 2005) e também o seu potencial para o controle da praga (GEDEN et al., 1998; CRAWFORD et al., 1998; ROHDE et al., 2006).

Alguns estudos também já foram realizados simulando as condições térmicas dos aviários, verificando-se que os isolados de B. bassiana foram mais sensíveis à temperatura de $32^{\circ} \mathrm{C}$ queMetarhiziumanisopliae (Metsch.) Sorok., sendo maior a eficiência dos fungos na temperatura de $26^{\circ} \mathrm{C}$ (ALEXANDre et al., 2006).

Em condições de campo, formulações de conídios de B. bassiana com farelo de milho, contendo 8,45'10 conídios/g, foram aplicadas diretamente sobre a cama do aviário e verificou-se que duas aplicações semanais não controlaram os cascudinhos, sugerindo a necessidade de estudos complementares GEDEN; STEINKRAUS, 2003).

Assim, este trabalho foi realizado com o objetivo de comparar diferentes aspectos envolvidos na definição de uma estratégia de utilização do fungoBeauveria bassiana no manejo das populações de cascudinho $A$. diaperinus em aviários comerciais.

\section{MATERIAL E MÉTODOS}

Foram utilizados insetos provenientes do campo devido à grande quantidade de indivíduos necessários, que foram aclimatados em laboratório por 5 a 7 dias, em recipientes plásticos de $50 \mathrm{~L}$ com tampa, juntamente com cama usada do aviário e ração para frango, preenchendo até $30 \%$ da capacidade do recipiente. Para utilização nos bioensaios, foram selecionadas larvas de terceiro e quarto ínstares (aproximadamente $1 \mathrm{~cm}$ de comprimento) e adultos com boa mobilidade (FrANCISCO; PRADO, 2001).

Utilizou-se o isolado UNIOESTE2 de B. bassiana, mantido na coleção da Unioeste, por apresentar elevada virulência (RoHDE et al., 2006), obtido de larvas de cascudinho coletadas em um aviário em Cascavel.

O fungo foi multiplicado em meio de cultura para esporulação (ALVES et al., 1998), incubado a $25 \pm 1^{\circ} \mathrm{Ce}$ fotofasede14horas, por10 dias atéplena conidiogênese. Os conídios foram recolhidos e utilizados para produção do fungo em arroz pré-cozido (Alves;PEREIRA, 1989, 1998), para posterior utilização nos bioensaios.

Comparação do tipo de aplicação. Prepararam-se uma suspensão comágua destilada+Tween $80(0,01 \%)$ (1 x $10^{8}$ conídios/mL) e formulação em pó com conídios+amido puro (PA) $\left(1 \times 10^{9}\right.$ conídios/g). Os experimentos foram realizados com cinco repetições de 15 insetos para cada estágio de vida (larva eadulto) separadamente, tendo-se como base concentrações utilizadas em estudos anteriores (STEINKRAUs et al., 1991; GEDEN et al., 1998).

Os insetos foram imersos em $1 \mathrm{~mL}$ da suspensão de conídios ou misturados a 0,1 g da formulação em pó, em copos plásticos, agitando-se por 15 segundos. As larvas foram individualizadas em caixas acrílicas de 3,0 cm de diâmetro, para evitar o canibalismo, e os adultos colocados em placas de Petri $(9 \mathrm{~cm}$ de diâmetro), todas com o fundo recoberto com papel-filtro e ração esterilizada para aves. Nas testemunhas, os insetos foram tratados com água destilada esterilizada + Tween $80(0,01 \%)$ ou amido puro. Todo o conjuntofoimantidoem potes plásticos com ofundo recoberto por espuma de poliuretano umedecida, incubados a $25 \pm 1^{\circ} \mathrm{C}$ e fotofase de 12 horas.

A avaliação foi feita após 5 e 10 dias do tratamento, e os insetos mortos foram imersos em álcool $70 \%$ e água destilada por 15 segundos e incubados em câmara úmida, nas condições descritas anteriormente, para confirmação da mortalidade pelo fungo, a qual foi acompanhada por até 10 dias, a partir da avaliação de mortalidade.

Estimativa da concentração letal média $\left(\mathrm{CL}_{50}\right)$. Foram preparadas cinco repetições de 15 insetos para cada estágio de vida (larva e adulto), separadamente. Cada grupo de insetos foi acondicionado em copos descartáveis de $10 \mathrm{~mL}$, e recebeu $0,1 \mathrm{~g}$ da formulação PA nas concentrações de $1 \times 10^{7}, 5 \times 10^{7}, 1 \times 10^{8}, 5 \times 10^{8}$, $1 \times 10^{9}$ conídios $/ g$, agitando-se por 15 segundos. As testemunhas receberam amido puro e, após o tratamento, os insetos foram mantidos nas mesmas condições descritas anteriormente.

Estimativa do tempo letal médio $\left(\mathrm{TL}_{50}\right)$. Foram preparadas cinco repetições com 15 insetos para cada dia de avaliação e para cada estágio de vida (larva e adulto). Os insetos foram tratados como descrito no experimento anterior, utilizando a formulação PA $(1 \mathrm{x}$ $10^{8}$ conídios/g). Os insetosforamacondicionadoscomo descrito anteriormente e a avaliação de mortalidade foi efetuada até o 10 dia após a inoculação, observando- 
se diariamente um conjunto de cinco repetições para o tratamento com fungo e cinco repetições para o tratamento controle, as quais foram descartadas, possibilitando a avaliação de amostras independentes. Os insetos mortos foram retirados esubmetidos ao mesmo tratamento descrito anteriormente.

Influência do tipo de solo, cobertura e local de aplicação do fungo. Foram utilizados os tratamentos constantes da Tabela 1, sendo empregados dois tipos de solo: solo de corte de barranco (Latossolo roxo distrófico A) de área sem qualquer atividade agrícola, onde, provavelmente, são encontrados baixos níveis de resíduos de produtos químicos, e solo proveniente do interior de aviários de frangos de corte, o qual certamente apresenta maiores teores de resíduos químicos, orgânicos e microrganismos, próprios do manejo do aviário. Ambos os solos foram peneirados (malha $4 \mathrm{~mm}$ ) e, em seguida, determinou-se a sua umidade pelo método gravimétrico.Quando necessário acrescentou-se água destilada, visando padronizar a umidade das amostras em aproximadamente $22 \%$, tendo como base a umidade do solo no interior doaviário, previamentedeterminada gravimetricamente.

Em seguida, $500 \mathrm{~g}$ de cada solo foram acondicionadas em potes plásticos com tampa $(10 \times 13 \times 17 \mathrm{~cm}$ - L:A:C), formando uma camada de, aproximadamente, $3 \mathrm{~cm}$ de profundidade e então liberaram-se 20 larvas ou 20 adultos no centro de cada recipiente (nos seus respectivos tratamentos). Após duas horas, polvilhou-se uniformemente na superfície do solo a formulação em pó $\left(1 \times 10^{10}\right.$ conídios $\left./ \mathrm{m}^{2}\right)$.

Outroconjuntodepotes recebeu somentecamanova ou usada, com aplicação da formulação em pó sobre ou abaixo destes substratos, de forma a simular os aviários com piso de cimento. Em seguida, cada recipiente recebeu 5 g de ração esterilizada para aves e para os tratamentos com cama (nova ou usada), e a ração foi distribuída logo após o solo ser tratado. Na testemunha, polvilhou-se amido puro de acordo com o local de aplicação.Para todos os tratamentosforam feitasquatro repetições com 20 larvas e 20 adultos separadamente.

Os potes foram mantidos a $25 \pm 1^{\circ}$ Ceescotofase de $24 \mathrm{~h}$, por 10 diase, para manter a umidade no interior de todos eles, pulverizaram-se $5 \mathrm{~mL}$ de água destilada, a cada 2 dias. Decorrido o período, fez-se a avaliação examinando-se todo o conteúdo dos recipientes. Os insetos mortos foram desinfectados em álcool $70 \%$ e água destilada por 15 segundos, sendo posteriormente incubados em câmara úmida, nas condições descritas anteriormente, para confirmação de mortalidade pelo fungo, sendo verificados por até 10 dias.

Efeito do solo e da cama de aviário na persistência e eficiência do fungo B. bassiana. Foramadotados os tratamentos: cama usada de aviário peneirada na forma natural (substrato A); cama usada autoclavada (substrato B) e solo de aviário (5 cm de profundidade) (substrato C). Os diferentes tratamentos foram acondicionados em potes plásticos de $500 \mathrm{~mL}$, formando camadas de $2 \mathrm{~cm}$ de profundidade. Cada grupo de 15 larvas ou adultos foi considerado uma repetição e foram inoculados em copos plásticos descartáveis de $10 \mathrm{~mL}$, contendo $0,1 \mathrm{~g}$ da formulação PA $\left(1 \times 10^{8}\right.$ conídios), sob agitação por 15 segundos. As respectivas testemunhas receberam amido puro. Após a inoculação, os insetos foram transferidos para potes contendo os diferentes substratos e mantidos a $25 \pm 1^{\circ}$ $\mathrm{C}$ e fotofase de 12 horas.

As avaliações foram realizadas após a aplicação e 12, 24, 48, 72 horas, retirando-se ao acaso quatro recipientes de cada tratamento, cada um considerado uma repetição. Os insetos mortos foram transferidos para câmara úmida, conforme descrito anteriormente, e os vivos transferidos para placas de Petri com ração esterilizada para aves, onde permaneciam por mais 10 dias sob observação diária, descartando-se os recipientes avaliados.

Tabela 1 - Tratamentos utilizados no estudo da influência do tipo de solo, cobertura e local de aplicação do fungo $B$. bassiana para controle de $A$. diaperinus, em laboratório.

\begin{tabular}{ll}
\hline Tratamentos com solo & Tratamentos sem solo \\
\hline Solo barranco & Cama nova (aplicação sobre) \\
Solo aviário & Cama nova (aplicação abaixo) \\
Solo barranco+cama nova & Cama usada (aplicação sobre) \\
Solo aviário+cama nova & Cama usada (aplicação abaixo) \\
Solo barranco+cama usada & Cama nova (controle) \\
Solo aviário+cama usada & Cama usada (controle) \\
Solo barranco (controle) & \\
Solo aviário (controle) & \\
Solo barranco+cama nova (controle) & \\
Solo aviário+cama nova (controle) & \\
Solo barranco+ cama usada (controle) & \\
Solo aviário+ cama usada (controle) & \\
\hline
\end{tabular}


Procedimentos para análise dos diferentes experimentos. Osdados de $\mathrm{CL}_{50} \mathrm{eTL}_{50}$ foram estimados pelo programa Polo-PC. O delineamento experimental foi inteiramentecasualizadoea análise estatística fatorial, com dados transformados em arcsen $\sqrt{\frac{x}{100}}+0,5$, foram submetidas à ANAVA, e as médias comparadas pelo teste de Tukey $(\mathrm{P} \leq 0,05)$, utilizando o programa estatístico SISVAR (FERREIRA, 1992).

\section{RESULTADOS E DISCUSSÃO}

Comparação do tipo de aplicação. A mortalidade confirmada delarvase adultos com a formulaçãoPA foi, respectivamente, $83,3 \mathrm{e} 33,4 \%$, significativamentemaior quea obtida comofungonaforma desuspensãoaquosa (18,7 para larvas e $12 \%$ para adultos) (Tabela 2).

Considerando-se que a germinação do conídio e conseqüente produção de enzimas para degradar a cutícula iniciam-se após a sua adesão no hospedeiro, a diferença nos resultados observados nos dois modos de aplicação (PA e líquido) pode ser atribuída à uma provável alteração na capacidade de adesão, por mudanças no potencial eletrostático dos conídios pela formulação aquosa (BIDOCHKA; KHACHATOURIANS, 1990). Substâncias como espalhante adesivo ou mesmo a água podemalterar as características hidrofóbicas dos conídios, comprometendo o reconhecimento da cutícula do hospedeiro (Boucias et al., 1988).

Embora SteinKRaus et al. (1991) tenham relatado que a formulação em pó de conídios de $B$. bassiana tenha sido menos efetiva que a suspensão aquosa, sobre larvas e adultos do cascudinho, quando aplicadas diretamente sobre os insetos, os mesmos autores observaram que a formulação em pó foi mais eficiente no tratamento da cama. Além disso, em experimento semelhante, GEDEN et al. (1998), tratando-se o solo ao invés da cama, verificaram a mesma tendência nos resultados.

O fato da preparação em pó resultar em maior mortalidade dos insetos, tanto na cama como no solo, confirma observações de FeNG et al. (1994) e BATISTA FILHO etal. (1998), segundo os quais as formulações em pó à base de conídios de fungos entomopatogênicos apresentam melhor dispersão na aplicação, além de maior viabilidade no armazenamento e são, geralmente, muito mais eficientes no controle dos insetos-alvo.

Estimativa da concentração letal média $\left(\mathrm{CL}_{50}\right)$ e Estimativa do tempo letal médio $\left(\mathrm{TL}_{50}\right)$. Foram obtidos, para larvas e adultos, valores de $\mathrm{CL}_{50}$ de $2,41 \times 10^{7}$ e 5,33 x $10^{7}$ conídios $/ g$, respectivamente e $\mathrm{TL}_{50}$ de 4,7 e 5,3 dias respectivamente (Tabela 3), confirmando que as larvas são mais suscetíveis ao fungo e morrem mais rapidamente (ROHDE et al., 2006).

GEDEN et al. (1998), comparando a eficiência de preparações líquidas e em pó de $B$. bassiana para cascudinho, em laboratório, obtiveram $\mathrm{CL}_{50}$ de 6,06x $10^{4}$ conídios/mL e 7,5 x $10^{8}$ conídios/g, respectivamente. Os resultados, superiores aos aqui obtidos, para a formulação PA, podem ser devido à maior virulência do isolado utilizado no presente trabalho.

Influência do tipo de solo, cobertura e local de aplicação do fungo. A menor mortalidade pode estar relacionada com um efeito antagônico do solo e da cama sobre ofungo ou a um comportamento delimpeza

Tabela 2 - Mortalidade média confirmada de larvas e adultos de $A$. diaperinus nos diferentes tratamentos com e sem $B$. bassiana.

\begin{tabular}{lrr}
\hline Tratamentos & \multicolumn{2}{c}{ Porcentagem de mortalidade } \\
\cline { 2 - 3 } & \multicolumn{1}{c}{ Larvas } & Adultos \\
\hline Pó seco (conídios + amido) & $81,3 \pm 3,24 \mathrm{a}$ & $33,4 \pm 1,75 \mathrm{a}$ \\
Suspensão aquosa (conídios + água) & $18,7 \pm 2,32 \mathrm{~b}$ & $12,0 \pm 1,21 \mathrm{~b}$ \\
Controle (amido) & $0,0 \pm 0,00 \mathrm{c}$ & $0,0 \pm 0,00 \mathrm{c}$ \\
Controle (água) & $0,0 \pm 0,00 \mathrm{c}$ & $0,0 \pm 0,00 \mathrm{c}$ \\
\hline
\end{tabular}

Médias seguidas de mesma letra, na coluna, não diferem estatisticamente pelo teste de Tukey $(\mathrm{P} \leq 0,05)$

Tabela 3 - $\mathrm{CL}_{50}$ (conídios/g) e $\mathrm{TL}_{50}$ (dias) para larvas e adultos de A. diaperinus tratados com formulação em pó (conídios + amido) de B. bassiana.

\begin{tabular}{llcrr}
\hline Estágio & Equação de regressão & $\mathrm{CL}_{50}\left(\mathrm{IC}_{0,05}\right)^{1}$ & $\mathrm{n}$ & $X^{2}$ \\
\hline Larva & $\mathrm{y}=6,481+2,012^{*} \log \mathrm{x}$ & $2,41 \times 10^{7}\left(1,36 \times 10^{7} 3,44 \times 10^{7}\right)$ & 75 & 8,9 n.s. \\
Adulto & $\mathrm{y}=5,688+1,920^{*} \log \mathrm{x}$ & $5,33^{\prime} 10^{7}\left(3,91 \times 10^{7}-6,56 \times 10^{7}\right)$ & 75 & $7,3^{\text {n.s. }}$ \\
\hline & & $\mathrm{TL}_{50}\left(\mathrm{IC}_{0,05}\right)$ & & \\
\hline Larva & $\mathrm{y}=8,656+1,620^{*} \log \mathrm{x}$ & $4,7(4,1-5,3)$ & 75 & $3,5^{\text {n.s. }}$ \\
Adulto & $\mathrm{y}=8,841+1,814^{*} \log \mathrm{x}$ & $5,3(4,4-6,1)$ & 75 & $3,8^{\text {n.s. }}$ \\
\hline
\end{tabular}

${ }^{1}$ Concentração de conídios/g da mistura com amido (1 x 10 conídios/g). 
doinseto, retirando os conídios de sua cutícula através do caminhamento na cama por fricção no substrato.

Neste sentido, maior mortalidade de larvas de Diaprepes abbreviatus L. porMetarhizium anisopliae eB. bassiana foi observada quando as larvas foram tratadas por doses subletais de imidacloprid e diminuíram sua movimentação no solo, sendo observado, posteriormente, que as micoses em $D$. abbreviatusestão bastante correlacionadas com a mobilidade do inseto no solo (Quintela; McCoy, 1998).

Para larvas não se observaram diferenças significativas da mortalidade quanto ao tipo de solo sozinho e com cama nova, somente sendo observadas diferenças para cama usada, com menor mortalidade para o solo de barranco $(7,7 \%)$. Contudo, a menor tendência de mortalidade de larvas neste tratamento levanta a hipótese de que neste tipo de solo houve uma maior movimentação das larvas no seu interior promovendo uma maior remoção dos por fricção com as partículas de solo, sendo que esta hipótese deve ser testada em estudos futuros.
A mortalidade confirmada de larvas foi estatisticamente superior no tratamento com cama nova, no qual a formulação foi aplicada tanto em sua superfície quanto abaixo. Contudo, ocorreu mortalidade de adultos somente no tratamento com aplicação sob a cama nova. Em todos os tratamentos em que se utilizou a cama usada, tanto para larvas quanto para insetos adultos, não houve mortalidade confirmada (Tabela 5).

Tal como observado anteriormente, a mortalidade sobre larvas foi maior do que a de adultos. A diferença de mortalidade entre a cama nova e usada decorre provavelmente da inativação dos conídios por desinfetantes e antibióticos utilizados na criação dos frangos e limpeza dos aviários, bem como da ação antagonista de microrganismos presentes na cama.

STEINKRAUS et al. (1991) e CRAWFORD et al. (1998) também observaram a redução na eficiência do fungo no controle do cascudinho quando aplicado na cama usada, em campo, atribuindo a sua temperatura como fator desativador de B. bassiana.

Tabela 4 - Mortalidade média confirmada de larvas e adultos de A. diaperinus em diferentes substratos tratados com formulação em pó de conídios de B. bassiana.

\begin{tabular}{lrr}
\hline Tratamentos & \multicolumn{2}{c}{$\%$ de mortalidade confirmada } \\
\cline { 2 - 3 } & \multicolumn{1}{c}{ Larva } & Adulto \\
\hline Solo barranco & $37,5 \pm 2,34$ & $55,0 \pm 1,33 \mathrm{a}$ \\
Solo aviário & $28,7 \pm 2,21 \mathrm{ab}$ & $13,7 \pm 1,14 \mathrm{c}$ \\
Solo barranco + cama nova & $15,0 \pm 1,75 \mathrm{bc}$ & $10,3 \pm 0,96 \mathrm{c}$ \\
Solo aviário + cama nova & $31,2 \pm 3,22 \mathrm{ab}$ & $0,0 \pm 0,00 \mathrm{~d}$ \\
Solo barranco + cama usada & $7,7 \pm 0,62 \mathrm{c}$ & $26,3 \pm 1,58 \mathrm{~b}$ \\
Solo aviário + cama usada & $30,0 \pm 1,74 \mathrm{ab}$ & $11,5 \pm 0,87 \mathrm{c}$ \\
Solo barranco (controle) & $0,0 \pm 0,00 \mathrm{~d}$ & $0,0 \pm 0,00 \mathrm{~d}$ \\
Solo aviário (controle) & $0,0 \pm 0,00 \mathrm{~d}$ & $0,0 \pm 0,00 \mathrm{~d}$ \\
Solo barranco + cama nova (controle) & $0,0 \pm 0,00 \mathrm{~d}$ & $0,0 \pm 0,00 \mathrm{~d}$ \\
Solo aviário + cama nova (controle) & $0,0 \pm 0,00 \mathrm{~d}$ & $0,0 \pm 0,00 \mathrm{~d}$ \\
Solo barranco + cama usada (controle) & $0,0 \pm 0,00 \mathrm{~d}$ & $0,0 \pm 0,00 \mathrm{~d}$ \\
Solo aviário + cama usada (controle) & $0,0 \pm 0,0 \mathrm{~d}$ & $0,0 \pm 0,00 \mathrm{~d}$ \\
\hline
\end{tabular}

Médias $( \pm \mathrm{EP})$ seguidas de mesma letra minúscula, na coluna, não diferem estatisticamente pelo teste de Tukey $(\mathrm{P} \leq 0,05)$.

Tabela 5 - Mortalidade média confirmada de larvas e adultos de A. diaperinus em substratos tratados em diferentes locais com formulação em pó de conídios de B. bassiana.

\begin{tabular}{|c|c|c|}
\hline \multirow[t]{2}{*}{ Tratamentos } & \multicolumn{2}{|c|}{ Porcentagem de mortalidade confirmada } \\
\hline & Larva & Adulto \\
\hline \multicolumn{3}{|l|}{ Aplicação sobre } \\
\hline Cama nova & $58,7 \pm 6,42 a$ & $0,0 \pm 0,00 \mathrm{~b}$ \\
\hline Cama usada & $0,0 \pm 0,00 \mathrm{c}$ & $0,0 \pm 0,00 \mathrm{~b}$ \\
\hline \multicolumn{3}{|l|}{ Aplicação abaixo } \\
\hline Cama nova & $38,7 \pm 3,87 b$ & $16,3 \pm 1,40 a$ \\
\hline Cama usada & $0,0 \pm 0,00 c$ & $0,0 \pm 0,00 \mathrm{~b}$ \\
\hline \multicolumn{3}{|l|}{ Controle } \\
\hline Cama nova & $0,0 \pm 0,00 c$ & $0,0 \pm 0,00 \mathrm{~b}$ \\
\hline Cama usada & $0,0 \pm 0,00 \mathrm{c}$ & $0,0 \pm 0,00 b$ \\
\hline
\end{tabular}

Médias( $\pm E P)$ seguidas de mesma letra minúscula, na coluna, não diferem estatisticamente pelo teste de Tukey $(\mathrm{P} \leq 0,05)$. 
Estes dados mostram que no desenvolvimento de uma estratégia de controle do cascudinho com $B$. bassiana, esta deverá ser aplicada preferencialmente antes do alojamento das aves.

Efeito do solo e da cama de aviário na persistência e eficiência do fungo $\boldsymbol{B}$. bassiana. Observou-se que o solo de aviário foi o mais prejudicial à ação do fungo, além de reduzir a mortalidade inicial para larvas, tal como observado para cama usada autoclavada. Contudo, a mortalidade final de larvas na cama usada autoclavada ou não, igualou-se.

Estes dados podem estar relacionados com os efeitos químicos ou físicos que promovem a redução. Provavelmente, quando os físicos atuam sozinhos, como no caso da cama autoclavada, são mais lentos, mas também importantes pelo valor final da redução na mortalidade (Tabela 6).

Para os adultos, ocorreu maior e mais rápida diminuição da mortalidade no tratamento cama usada, o que pode estar relacionado com uma menor atividade e conseqüente movimentação destes insetos neste substrato. Entretanto, em todos os casos, o aumento do tempo de contato dos insetos (larvas e adultos) com os substratos ocasionou diminuição da mortalidade, independente do substrato utilizado (Tabela 6).

Expondo-se larvas e adultos do cascudinho recém-tratados por imersão em suspensão de conídios de $B$. bassiana à cama nova e usada de aviário, ALEXANDRE et al. (2006) também verificaram que a eficiência do fungo foi significativamente afetada quando em contato com a cama usada (2,3 e 8,9\% de mortalidade de larvas e adultos, respectivamente), em relação aos valores obtidos nos recipientes com cama nova $(15,7$ e $66,7 \%$, para larvas e adultos) ou apenas ração para aves (93,3 e 31,1\% com larvas e adultos).

Ressalta-se que a amônia está presente em grande quantidade na cama usada de aviários e esta possui efeito fungistático, afetando a germinação de esporos de diferentes espécies de fungos (BACON, 1985).

Além de metabólitos, actinomicetos e outras bactérias podem agir como agentes inibidores da germinação de B. bassiana (WARTENBERG; FREUND, 1961), podendo estar presentes tanto no solo como na cama usada do aviário, sendo que observações semelhantes foram feitas por MoINoJunior; Alves (1999), em um estudo de controle microbiano de cupins.

Outra possibilidade é que a cama usada é formada por partículas de madeira de menor granulometria em relação à cama nova, já que o caminhamento contínuo das aves provoca fragmentação da maravalha (KoONet al.,1994). Osinsetos tratados, liberados emseguidanos recipientes, por apresentarem movimentação contínua, mantêm contato com tais partículas que, provavelmente, removeram os conídios aderidos aos insetos antes mesmo que germinassem.

Apesar de serem encontrados muitos insetos mortos no solo dos aviários com sinais de fungo (Alves et al., 2004; Alvesetal.,2005), da mesma forma o solo pode apresentar efeito fungistático, dependendo do seu tipo, em função do seu $\mathrm{pH}$, textura, quantidade de matéria orgânica ou carbono (GRODEN; LOCKWOOD, 1991).

A remoção do fungo da superfície dos insetos foi também comprovada em trabalhos realizados com cupins subterrâneos, tanto pelo comportamento dos insetos se limparem ou como resultado do deslocamento dos insetos pelo substrato (NEvEs;Alves, 2000).

Tabela 6 - Mortalidade média confirmada e redução em relação ao tempo 0, de larvas e adultos de A. diaperinus tratados com formulação em pó seco de B. bassiana, expostos em três substratos por tempos diferentes.

\begin{tabular}{|c|c|c|c|c|c|c|}
\hline \multirow{3}{*}{$\begin{array}{l}\text { Avaliação } \\
\text { (horas) } \\
\text { Larva }\end{array}$} & \multicolumn{6}{|c|}{ Substratos } \\
\hline & \multicolumn{2}{|c|}{ A (cama usada natural) } & \multicolumn{2}{|c|}{ B (cama usada autoclavada) } & \multicolumn{2}{|c|}{ C (solo de aviário natural } \\
\hline & Confirmada & Redução $^{1}$ & Confirmada & Redução $^{1}$ & Confirmada & Redução ${ }^{1}$ \\
\hline 0 & $73,3 \pm 2,41 \mathrm{Aa}$ & - & $73,3 \pm 2,41 \mathrm{Aa}$ & - & $38,3 \pm 2,41 \mathrm{Ab}$ & - \\
\hline 12 & $57,3 \pm 1,97 \mathrm{Bb}$ & 21,8 & $64,0 \pm 4,10 \mathrm{Ba}$ & 12,7 & $16,7 \pm 2,26 \mathrm{Cc}$ & 56,4 \\
\hline 24 & $44,0 \pm 3,30 \mathrm{Cb}$ & 40,0 & $53,3 \pm 3,35 \mathrm{Ca}$ & 27,3 & $20,0 \pm 1,60 \mathrm{Bc}$ & 47,8 \\
\hline 48 & $40,0 \pm 4,14 \mathrm{Cb}$ & 45,4 & $45,3 \pm 2,88 \mathrm{Da}$ & 38,2 & $18,3 \pm 3,34 \mathrm{BC} \mathrm{C}$ & 52,2 \\
\hline 72 & $44,0 \pm 1,08 \mathrm{Ca}$ & 40,0 & $44,0 \pm 2,99 \mathrm{Da}$ & 40,0 & $3,3 \pm 6,40 \mathrm{Db}$ & 91,4 \\
\hline Adulto & Confirmada & Redução $^{1}$ & Confirmada & $\overline{\text { Redução }}{ }^{1}$ & Confirmada & Redução $^{1}$ \\
\hline 0 & $40,0 \pm 1,46 \mathrm{Ab}$ & - & $40,0 \pm 1,46 \mathrm{Ab}$ & - & $66,7 \pm 1,96 \mathrm{Aa}$ & - \\
\hline 12 & $34,7 \pm 3,65 \mathrm{Ba}$ & 13,250 & $29,3 \pm 2,75 \mathrm{Bb}$ & 26,8 & $26,6 \pm 3,30 \mathrm{Cb}$ & 60,1 \\
\hline 24 & $25,3 \pm 2,17 \mathrm{Cb}$ & 36,750 & $27,3 \pm 2,17 \mathrm{Bb}$ & 31,8 & $38,3 \pm 3,53 \mathrm{Ba}$ & 42,6 \\
\hline 48 & $26,7 \pm 2,53 \mathrm{Cb}$ & 33,250 & $30,7 \pm 4,60 \mathrm{Ba}$ & 23,3 & $25,0 \pm 3,20 \mathrm{Cb}$ & 62,5 \\
\hline 72 & $25,3 \pm 3,38 \mathrm{Ca}$ & 36,750 & $20,0 \pm 2,07 \mathrm{Ca}$ & 50,0 & $23,4 \pm 2,96 \mathrm{Ca}$ & 64,9 \\
\hline
\end{tabular}

${ }^{1}$ Redução de mortalidade em relação à avaliação inicial (0 horas).

Média $( \pm E P)$ seguidas de mesma letra maiúscula, na coluna, e minúsculas, nas linhas, não diferem estatisticamente pelo teste de Tukey $(\mathrm{P} \leq 0,05)$. 
Como forma de minimizar os efeitos de fatores físicoquímicosoumicrobiológicos dosubstratosobreofungo, GEDEN et al. (1998) sugeriram a utilização de estratégias nas quais os insetos sejam atraídos para fora da cama com armadilhas impregnadas com fungo ou que sejam testadas aplicações na superfície do solo, após os procedimentos de troca da cama elimpeza do aviário. Assim, novos estudos integrando estes eoutros conhecimentos sobre os fatores do substrato serão fundamentais para determinar quais estratégias de controle podem ser empregadas para utilização de fungos entomopatogênicos em aviários de frangos de corte.

Considerando todos os fatores expostos acima conclui-se que, na definição de uma estratégia de controle de populações de $A$. diaperinuscom B.bassiana, a formulação tipo pó mostrou-se mais promissora e eficiente tanto para adultos como para larvas. Entretanto, devem ser desenvolvidas formulações e/ou estratégias que possam melhorar ainda mais esta eficiência bem como a persistência e a viabilidade.

Neste sentido, verificou-se, em laboratório, que a terra de diatomácea apresenta atividade inseticida contra o cascudinho (Alves et al.,2006), sendo que sua associação com fungos entomopatogênicos já foi avaliada com bons resultados para outros insetos (LORD, 2001; Akbar et al., 2004) e também para o cascudinho (Oliveira et al., comunicação pessoal).

Além disso, os dados indicam que se deve usar o fungo preferencialmente para larvas e na troca da cama, antes da instalação do novo lote, pois a cama usada diminui a viabilidade do fungo provavelmente por efeito antagônico.

Esta estratégia deve também ser utilizada quando se considera a cobertura e o local de aplicação bem comoda persistência dofungonos diferentes substratos. Assim, são agora necessários novos estudos em aviários para testar os resultados aqui obtidos e a sua inserção no manejo das populações de $A$. diaperinus.

\section{REFERÊNCIAS}

AKBAR, W.; LORD, J.C.; NECHOLS, J.R.; HOWARD, R.W. Diatomaceous earth increases the Efficacy of Beauveria bassiana against Tribolium castaneum Larvae and increases conidia attachment. Journal of Economic Entomology, v.97, p.273-280, 2004.

Alexandre, T.M.; Alves, L.F.A.; Neves, P.M.O.J.; Alves, S.B. Efeito da temperatura e substrato sobre Beauveria bassiana e Metarhizium anisopliae e sua relação no controle do cascudinho (Alphitobius diaperinus) (Panzer) (Coleoptera: Tenebrionidae). Neotropical Entomology, v.35, n.1, p.75-82, 2006.

ALVES, L.F.A.; BUSARELLO, G.D.; OLIVEIRA, D.G.P.; ALVES, S.B. Ação da terra de diatomácea contra adultos do cascudinho Alphitobius diaperinus (Panzer, 1797) (Coleoptera: Tenebrionidae). Arquivos do Instituto Biológico, São Paulo, v.73, n.1, p.115-118, 2006.

ALVES, L.F.A.; BRESSAN, D.F.; ALVES, V.S.; NEVES, P.M.O.J.; Alves, S.B. Ocorrência de Metarhizium anisopliae (Metsch.) Sorok. (Moniliales: Moniliaceae) em adultos de cascudinho (Alphitobius diaperinus) (Panzer) (Coleoptera: Tenebrionidae), em aviários comerciais em Cascavel, PR. Neotropical Entomology, v.33, n.6, p.793-795, 2004.

ALVES, L.F.A.; GASSEN, M.H.; PINTO, F.G.S.; NEVES, P.M.O.J.; ALVES, S.B. Ocorrência natural de Beauveria bassiana (Bals.) Vuilleman (Moliniales: Moniliaceae) sobre o cascudinho (Alphitobius diaperinus) (Panzer) (Coleoptera: Tenebrionidae), em avário comercial em Cascavel, PR. Neotropical Entomology, v.34, n.3, p.507510, 2005.

ALVES, S.B.; ALMEIDA, J.E.M.; MOINO JUNIOR, A.; ALVES, L.F.A. Técnicas de laboratório. In: ALVES, S.B. (Ed.). Controle microbiano de insetos. 2. ed. Piracicaba: FEALQ, 1998. p.637-712.

ALVES, S.B.; PEREIRA, R.M. Produção de Metarhizium anisopliae (Metsch.) Sorok. e Beauveria bassiana (Bals.) Vuill. em bandejas. Ecossistema, v.14, p.188-192, 1989.

ALVES, S.B.; PEREIRA, R.M. Produção de fungos entomopatogênicos. In: Alves, S.B. (Ed.). Controle microbiano de insetos. 2. ed. Piracicaba: FEALQ, 1998. p.845-870.

AXTELL, R.C.; ARENDS, J.J. Ecology and management of arthropod pests of poultry. Annual Review of Entomology, v. 35, p.101-126, 1990.

BACON, C.W. Effects of broiler litter volatiles and ammonia on fungal spore germination. Poultry Science, v.65, p.710-716, 1985.

BATISTA FILHO, A.; ALVES, S.B.; ALVES, L.F.A.; PEREIRA, R.M.; AUGUSTO, N.T. FORMULAÇÃO DE ENTOMOPATÓGENOS. IN: ALVES, S.B. (Ed.). Controle microbiano de insetos. 2. ed. Piracicaba: FEALQ, 1998. p.917-966.

BIDOCHKA, M.J.; KHACHATOURIANS, G.G. Identification of Beauveria bassiana extracellular protease as a virulence factor in pathogenecity toward the migratory grasshopper, Melanoplus sanguinipes. Journal Invertebrate Pathology, v.56, p.362-370, 1990.

BOUCIAS, D.G.; PENDLAND, J.C.; LATGE, J.P.

Nonspecific factors involved in attachment of entomopathogenic deuteromycetes to host insect cuticle. Applied Environmental Microbiology, v.54, p.1795-1805, 1988.

CHERNAKI-LEFFER, A.M. Dinâmica populacional, estimativa da resistência a inseticidas e alternativas de 
controle para o cascudinho Alphitobius diaperinus (Panzer, 1797) (Coleoptera: Tenebrionidae). 2004, 123f. Tese (Doutorado em Zoologia) - Departamento de Zoologia, Universidade Federal do Paraná, Curitiba, 2004.

CHERNAKI, A.M.; ALMEIDA, L.M. Exigências térmicas, período de desenvolvimento e sobrevivência de imaturos de Alphitobius diaperinus (Panzer) (Coleoptera: Tenebrionidae). Neotropical Entomology, v.30, n.3, p.365-368, 2001a.

CHERNAKI, A.M.; ALMEIDA, L.M. Morfologia dos estágios imaturos e do adulto de Alphitobius diaperinus (Coleoptera: Tenebrionidae). Revista Brasileira de Zoologia, v.18, n.2, p.351-363, 2001b.

CRAWFORD, P.J.; BROOKS, W.M.; ARENDS, J.J. Efficacy of field-isolated strains of Beauveria bassiana (Moniliales: Moniliaceae) as microbial control agents of the lesser mealworm (Coleoptera: Tenebrionidae). Journal of Economic Entomology, v.91, n.6, p.1295-1301, 1998.

DESPINS J.L.; AXTeLL, R.C. Feeding behavior and growth of broiler chicks fed larvae of the darkling beetle, Alphitobius diaperinus. Poultry Science, v.74, p.331-336, 1995.

DESPINS J.L.; AXTELL, R.C.; RIVES, D.V.; GUY, J.S.; FICKEN, M.D. Transmission of enteric pathogens of turkeys by darkling beetle larva (Alphitobius diaperinus). Journal of Applied Poultry Research, v.3, p.61-65, 1994.

FENG, M.G.; POPRAWSKI, T.J.; KHACHATOURAIS, G.G. Production, formulation and application of the entomopathogenic fungus Beauveria bassiana for insect control: currents status. Biocontrol Science and Technology, v.4, p.3-34, 1994.

FERREIRA, D.F. SISVAR (Sistema para análise de variância para dados balanceados). Lavras: UFLA, 1992. 79p.

FRANCISCO, O.; PRADO, A.P. Characterization of the larval stages of $A$. diaperinus (Panzer) (Coleoptera: Tenebrionidae) using head capsule width. Revista Brasileira de Biologia, v.61, p.125-131, 2001.

GEDEN, C.J.; ARENDS, J.J.; RUTZ, D.A.; STEINKRAUS, D.C. Laboratory evaluation of Beauveria bassiana (Moniliales: Moniliaceae) against the lesser mealworm, Alphitobius diaperinus (Coleoptera: Tenebrionidae), in poultry litter, soil, and a pupal trap. Biological Control, v.13, p.71-77, 1998.

GEDEN, C.J.; STEINKRAUS, D.C. Evaluation of three formulations of Beauveria bassiana for control of lesser mealworm and hide beetle in Georgia poultry houses. Journal of Economic Entomology, v.96, n.5, p.1602-1607, 2003.

GRODEN, E.; LOCKWOOD, J.L. Effects of soil fungistasis on Beauveria bassiana and its relationship to disease incidence in the Colorado Potato Beetle,
Leptinotarsa decemlineata, in Michigan and Rhode Island soils. Journal of Invertebrate Pathology, v.57, p.7-16, 1991.

KOON, J.L.; FLOOD JUNIOR, C.A.; MCCASKEY, T.A.; BREWER, R.N. Changes in Physical and Chemical Characteristics of Poultry Litter due to rotary tilling. Transactions of the ASAE, v.37, n.1, p.269-274, 1994.

LEORA SOFTWARE. Polo-PC: A user's guide to Probit Logit analysis. Berkeley: Leora Software, 1987. 22p. LORD, J.C. Desiccant Dust Synergize the effect of Beauveria bassiana (Hyphomycetes: Moniliales) on Stored-grain beetles. Journal of Economic Entomology, v.94, p.367-372, 2001.

MOINO JUNIOR, A.; ALVES, S.B. Efeito antagônico de Trichoderma sp. no desenvolvimento de Beauveria bassiana (Bals.) Vuill. e Metarhizium anisopliae (Metsch.) Sorok. Scientia Agricola, v.56, n.1, p.217-224, 1999.

NEVES, P.J.; ALVES, S.B. Selection of Beauveria bassiana (Bals.) Vuill. and Metarhizium anisopliae (Metsch) Sorok. strains for control of Cornitermes cumulans (Kollar). Brazilian Archives of Biology and Technology, v.43, n.4, p.373-378, 2000 .

QUINTELA, E.D.; MCCOY, C. Synergistic effect of imidacloprid and two entomopathogenic fungi on the behavior and survival of larvae of Diaprepes abbreviatus (Coleoptera: Curculionidae) in soil. Journal of Economic Entomology, v.91, p.110-122, 1998.

ROHDE, C.; ALVES, L.F.A.; BRESSAN, D.F.; NEVES, P.M.O.J.; SILVA, E.R.L.; ALVES, S. B.; ALMEIDA, J.E.M. Seleção de isolados de fungos para o controle do cascudinho Alphitobius diaperinus (Panzer) (Coleoptera: Tenebrionidae). Neotropical Entomology, v.35, n.2, p.231-240, 2006.

STEINKRAUS, D.C.; BROOKS, W.M.; GEDEN, C.G. Discovery of the neogregarine Farinocystis tribolii and an eugregarine in the lesser mealworm, Alphitobius diaperinus. Journal of Invertebrate Patology, v.59, p.203205, 1992.

STEINKRAUS, D.C.; GEDEN, C.J.; RUTZ, D.A. Susceptibility of lesser mealworm (Coleoptera: Tenebrionidae) to Beauveria bassiana: Effects of host stage, formulation, substrate and host passage. Journal of Medical Entomology, v.28, p.314-321, 1991.

WARTENBERG, H.; FREUND, K. Der Konservierungseffekt antibiotscher Mikrooganismen an Konidien van Beauveria bassiana (Bals.) Vuill. Zentralblatt für Bakteriologie, v.114, p.718-724, 1961.

Recebido em 17/1/07

Aceito em 18/3/08 